\title{
Comparative effectiveness of glycemic control in patients with type 2 diabetes treated with GLP-I receptor agonists: a network meta-analysis of placebo-controlled and active-comparator trials
}

\author{
This article was published in the following Dove Press journal: \\ Diabetes, Metabolic Syndrome and Obesity:Targets and Therapy \\ 29 March 2017 \\ Number of times this article has been viewed
}

\author{
Michelle E Orme' \\ Hiep Nguyen ${ }^{2}$ \\ Jackie $Y \mathrm{Lu}^{3}$ \\ Susan A Thomas ${ }^{3}$ \\ IICERA Consulting Ltd, Swindon, UK; \\ ${ }^{2}$ AstraZeneca, Wilmington, DE, \\ ${ }^{3}$ AstraZeneca, Fort Washington, \\ PA, USA
}

\begin{abstract}
Background: Clinical studies of patients with type 2 diabetes show that GLP-1 receptor agonists (GLP-1 RAs) improve glycemic control and promote weight loss. We conducted a Bayesian network meta-analysis (NMA) of placebo- and active-controlled randomized trials to assess the comparative effectiveness of liraglutide, albiglutide, dulaglutide, and exenatide twice daily and once weekly, with a focus on glycemic control.

Materials and methods: We searched Medline, Embase, and the Cochrane Library (up to December 2014) for core registration programs for US-approved GLP-1 RAs. Patients reaching an $A_{1 C}$ target of $<7 \%$ were analyzed with a binomial model and change in $A_{1 C}$ from baseline with a normal model. A covariate analysis assessed the impact of baseline $\mathrm{A}_{1 \mathrm{C}}$ and treatment background on outcomes.

Results: The base-case NMA used 23 trials reporting $A_{1 C}$ outcomes at $\sim 6$ month follow-up. The results, unadjusted and adjusted for baseline $\mathrm{A}_{1 \mathrm{C}}$, indicated that all GLP-1 RAs resulted in statistically significantly lower $\mathrm{A}_{1 \mathrm{C}}$ at follow-up compared with placebo. The odds of reaching the $<7 \%$ target were also significantly better compared with placebo. With dulaglutide, exenatide once weekly, and liraglutide, the absolute reduction in $\mathrm{A}_{1 \mathrm{C}}$ at 6 months was $0.9 \%-1.4 \%$, and was significantly better than exenatide twice daily. Albiglutide was not significantly different from exenatide twice daily. We estimate that $\sim 50 \%$ of patients will meet the $<7 \% \mathrm{~A}_{1 \mathrm{C}}$ target within 6 months of commencing GLP-1 RAs.
\end{abstract}

Conclusion: This was a comprehensive assessment of the comparative effectiveness of GLP-1 RAs and $\mathrm{A}_{1 \mathrm{C}}$ outcome. GLP-1 RAs are a viable addition to oral antidiabetes therapy, and dulaglutide, exenatide once weekly, and liraglutide are the most effective.

Keywords: type 2 diabetes, glucagon-like peptide-1-receptor agonists, GLP-1 RAs, network meta-analysis, comparative effectiveness

\section{Background}

Comparative effectiveness research (CER) in diabetes is increasingly important, given the wide range of treatment options available to patients with type 2 diabetes (T2D). Over the years, the goals of diabetes management have expanded beyond glycemic control to include the management of metabolic and cardiovascular comorbidities according to several international guidelines. ${ }^{1-5}$ Several newer classes of antihyperglycemic agents, including GLP-1 RAs and sodium-glucose cotransporter inhibitors, have been suggested to provide additional benefits, such as weight loss. Both the American Association of Clinical Endocrinologists guidelines and the American Diabetes
Correspondence: Hiep Nguyen AstraZeneca, 1800 Concord Pike B2B-I I2, Wilmington, DE 19850, USA $\mathrm{Tel}+$ I 215542335 I

Email hiep.nguyen@astrazeneca.com 
Association recommend a patient-centered approach to guide choice of pharmacological agents. ${ }^{2,6}$ Considerations include efficacy, cost, potential side effects, weight, comorbidities, hypoglycemia risk, and patient preferences. Both bodies recognize that GLP-1 RAs have robust $\mathrm{A}_{1 \mathrm{C}}$-lowering properties, are usually associated with weight loss and blood-pressure reductions, and are available in several formulations. The risk of hypoglycemia with GLP-1 RAs is low, and they reduce fluctuations in both fasting and postprandial states.

GLP-1 RAs are a growing class of glucose-lowering drugs that improve glucose homeostasis by enhancing the endogenous secretion of insulin induced by meal ingestion, inhibiting glucagon secretion, and slowing gastric emptying. Notably, they also suppress food intake and appetite, through central effects. ${ }^{7}$ Since the first GLP-1 RA was approved in 2005, the number of injectable agents in this class has increased from exenatide twice daily (EBID [exenatide bis in die]) to include liraglutide (Lira) once daily, exenatide once weekly (EQW [exenatide quaque week]), albiglutide (Albi) QW, and dulaglutide (Dula) QW.

Given the wide choices of GLP-1 RA agents, CER can be a useful tool to aid health care decision makers weigh up the benefits and harms associated with different treatment options. A common CER approach is to synthesize the available randomized controlled trial (RCT) evidence in a meta-analysis to provide a comprehensive view of the relative efficacy of the treatment options. The standard direct meta-analysis method is limited to evaluating the relative efficacy of treatments in a pairwise manner, where all the trials included in the direct meta-analysis compare the same intervention with the same control. Many trials are either placebo-controlled, include an active control that does not represent the current standard of care, or may not be comparable to the active arm in a treatment decision-making context. In the absence of head-to-head trials, indirect comparisons can be made using a common control arm to bridge the gap, provided that the randomized comparisons within each trial are preserved. ${ }^{8,9}$ Network meta-analysis (NMA), an extension of the standard meta-analysis methods, calculates the relative effects for all treatments in the evidence network in one simultaneous analysis. ${ }^{10-12}$ NMA is different from pairwise meta-analysis in the sense that there is not only one type of treatment comparison, but multiple treatment comparisons. Therefore, the NMA output provides a comprehensive evidence base that allows decision makers to compare the effects from any two treatments within the network, including the relative-effect estimates between treatments that have not been compared in head-to-head trials. NMAs can also provide more precise estimates of treatment differences than can be obtained from pairwise meta-analysis, since more of the data are used. ${ }^{10-12}$

There are several published meta-analyses evaluating the clinical profile of GLP-1 RAs; ${ }^{13-19}$ however, these analyses either had limited data for more recent US Food and Drug Administration-approved GLP-1 RAs, including QW formulations, did not apply the NMA methods to compare the relative efficacy of GLP-1 RAs, used a frequentist NMA method, or did not control for baseline $A_{1 C}$. Therefore, we performed a Bayesian NMA of placebo-controlled and activecontrolled randomized trials to assess the relative effect of Lira, Albi, Dula, EBID, and EQW, with a particular focus on glycemic control.

\section{Materials and methods Study selection and data sources}

We identified eligible studies by searching Medline, Embase, and the Cochrane Library from inception up to December 31, 2014, using pertinent keywords, and restricted our results to published RCTs in the English language. We included RCTs from core registration programs for all US-approved GLP-1 RAs. We included open-label and double-blind RCTs comparing one GLP-1 RA with another, at any dose or with a control (placebo, oral antihyperglycemic drugs), for adults with T2D. For inclusion, studies had to fulfill the following criteria: 1) placebo-controlled or active-comparator RCTs comparing EQW, EBID, Dula, Lira, or Albi in patients with T2D inadequately controlled with current therapy; 2) reported outcome of percentage of patients achieving $\mathrm{A}_{1 \mathrm{C}}<7 \%$ target; 3 ) provided mean change in $A_{1 C}$ from baseline with standard error or $95 \%$ confidence interval $[\mathrm{CI}]$; and 4 ) to be included in the base-case analysis, outcomes needed to be reported at 6-month follow-up (included range 24-32 weeks).

\section{Data extraction}

Data were from full-text publications for all GLP-1 RA RCTs used for product registration in the US. Data were extracted by two reviewers, and discrepancies resolved by consensus. For some studies, it was necessary to supplement the data extraction with information from clinical trial-registry records. ${ }^{20}$

\section{Outcome measurements and treatments}

The outcome data extracted included the percentage of patients with $\mathrm{A}_{1 \mathrm{C}}$ below target of $7 \%$ at follow-up and change from baseline in $A_{1 C}$ at follow-up. Change in $A_{1 C}$ from baseline was most commonly reported on a modified 
intent-to-treat basis where the population was defined as the set of patients who were randomized, received at least one dose of study medication, and had at least one postbaseline $\mathrm{A}_{1 \mathrm{C}}$ measurement. For the $\mathrm{A}_{1 \mathrm{C}}$ target outcome, trials reported the percentage of patients reaching treatment targets on an evaluable case basis, whereby only the subset of patients with $A_{1 C}>7 \%$ at baseline was evaluated.

For the NMA, we pooled data by GLP-1 RA agent: Albi, Dula, EBID, EQW, and Lira. GLP-1 RA treatment arms at unlicensed doses were not included. Other control arms were included in the evidence networks to preserve randomization, and these were pooled by treatment class as follows: placebo, DPP4 inhibitor, insulin, metformin, sulfonylurea, and thiazolidinedione.

\section{Meta-analyses}

The NMA approach was as per the UK's National Institute for Health and Care Excellence (NICE) Decision Support Unit recommendations for Bayesian NMA. ${ }^{21}$ This methodology is widely used for synthesizing clinical trial data for health-technology appraisal or regulatory purposes. ${ }^{22-24}$ The Bayesian statistical model applies Monte Carlo simulations, which converge the direct (A versus B) and indirect (A versus $\mathrm{C}, \mathrm{C}$ versus $\mathrm{B}$ ) evidence with the likelihood-effect estimate, and provides a modeled comparison between $A$ versus $B$ versus $\mathrm{C}$. The underlying assumption of this approach is that the comparator group for the interventions (ie, C) is similar among the indirect-comparison trials. Continuous outcomes were analyzed using a normal model with an identity link, and dichotomous outcomes were analyzed using a binomial model with logit link. Both fixed-effect and random-effect models were investigated. Fixed and random-effect models were fitted to the data via Bayesian Markov chain Monte Carlo methods using WinBUGs $1.4^{25}$ and were run in for a minimum of 100,000 iterations to ensure convergence. Subsequently, two chains of 100,000 were sampled from the posterior distributions. These samples were used to calculate the median/mean and the $95 \%$ credible interval $(\mathrm{CrI})$, which is the interval from the percentiles 2.5 to -97.5 . The CrI, distinct from the $\mathrm{CI}$, is the Bayesian equivalent of the frequentist $95 \% \mathrm{CI}$, and is used to assess statistically significant differences, which is consistent with the approach used by NICE in evaluating effectiveness data.

All results for the NMA are reported as medians with corresponding 95\% CrIs. Medians are presented as the best estimate for the central value, since means may be overly influenced by outliers. The pooled summary measure for continuous end points is weighted mean differences and odd ratios for binomial outcomes. An estimate of how well the predicted values fitted the observed data set was provided by the mean residual deviances (total residual deviance divided by number of data points), as well as the deviance information criteria (DIC) output from WinBUGs. ${ }^{21}$ Models with a good fit would have a total residual deviance close to the number of data points. The DIC is used to compare different models for the same likelihood and data, and the model with the lowest DIC was deemed to best predict a replicate data set of the same structure to that observed..$^{26,27}$ There were no major differences in DIC when comparing fixed-effect with random-effect models. Fixed-effect models assume that differences across trials do not impact on the treatment effects, and that variation in the outcomes reported are due to differences between patients within a trial. Random-effect models assume that variation in the outcomes reported are due both to differences between patients within a trial and differences across trials. ${ }^{28-30}$ Therefore, results from the random-effect NMA models have been presented in this paper, since these better take into account sources of uncertainty.

Covariate analyses were conducted to explore the effect of baseline $\mathrm{A}_{1 \mathrm{C}}$ and use of background treatment that may confound the $\mathrm{A}_{1 \mathrm{C}}$ end point. ${ }^{31}$ Previous meta-analyses have shown that there is a correlation between baseline $A_{1 C}$ and change in $A_{1 C}$ over follow-up. ${ }^{32}$ Therefore, a continuous study-arm level variable for baseline $A_{1 C}$ was included in the model, centered at the mean baseline $\mathrm{A}_{1 \mathrm{C}}$ across all study arms, the assumption being that the baseline $\mathrm{A}_{1 \mathrm{C}}$ has the same impact on effects across all treatments. In a further covariate analysis, a continuous study-arm level variable for percentage of patients on oral therapy as background (0-100\%) and a dummy-indicator variable for use of insulin as background treatment $(1$, insulin included in background; 0 , insulin not used as background treatment) were included to account for differences in background treatment. Note that covariate meta-analysis adjusts for differences between study arms, and as aggregated data are used in the covariate meta-analysis, the results should not be used to make predictions about individual patients.

The majority of studies reported outcomes at approximately 6-month follow-up. Some studies were of a longer duration, and a few studies did not provide sufficient endpoint data at 6-month follow-up in either the full-text publication or the clinical trial-registry record. For the base-case analysis, we used all studies that reported the outcomes of interest between 24 and 32 weeks of follow-up. A sensitivity analysis was conducted using all studies regardless of the follow-up time. 
In addition to the NMA, standard direct meta-analysis was also conducted in Stata version $14 .{ }^{33}$ We pooled studies using fixed- and random-effect models, using the randomeffect method of DerSimonian and Laird, where the estimate of between-study heterogeneity is taken from the fixed-effect Mantel-Haenszel or inverse-variance model. ${ }^{30,34}$ The direct meta-analysis was conducted to supplement the NMA results and to investigate potential inconsistencies between the direct and indirect estimates.

\section{Results}

In total, 29 GLP-1 RA core registration trials were identified covering 18,543 patients, which included seven trials for Albi, ${ }^{35-41}$ six trials for Dula, ${ }^{42-47}$ four trials for EBID, ${ }^{48-51}$ six trials for EQW, ${ }^{52-57}$ and six trials for Lira ${ }^{58-63}$ (Table S1). Seven of these trials provided head-to-head comparisons of GLP-1 RAs (Table 1). ${ }^{41,42,47,52,56,57,63}$ Given the results of the direct meta-analyses, different inferences can be made depending on which set of head-to-head trials is used, eg, Dula versus EQW via EBID controlled trials or via Lira controlled trials (Table 1). This demonstrates the need for an NMA to provide a comprehensive assessment of the comparative effectiveness of the GLP-1 RAs.

The base-case NMA consisted of 23 trials reporting outcomes at approximately 6-month follow-up (Figure 1, Network 1). The six trials excluded from the base case either reported data at 52 weeks (LEAD-3, HARMONY-1, 2, 4, and 5) or 104 weeks (HARMONY-3). The sensitivity analysis included all 29 trials (Figure 1, Network 2).

Across all analyses, the random-effect models had a better fit compared with the fixed-effect models in terms of DIC and average residual deviance. The covariate random-effect models, where treatment effects were adjusted for baseline $\mathrm{A}_{1 \mathrm{C}}$, had a similar fit to the unadjusted random-effect models. While baseline $A_{1 C}$ was not a statistically significant predictor of differences in treatment effects, the direction of effect indicates that study arms with a higher baseline $\mathrm{A}_{1 \mathrm{C}}$ will show a larger effect on $\mathrm{A}_{1 \mathrm{C}}$ compared with study arms with lower baseline $A_{1 C}$. Baseline $A_{1 C}$ does not appear to be a confounding factor in analysis of the $<7 \%$ target end point. However, based on model fit and observations from other analyses, ${ }^{32}$ the random-effect model adjusted for baseline $\mathrm{A}_{1 \mathrm{C}}$ is likely to provide the most robust results, since this takes into account some of the heterogeneity between studies.

Table 2 shows the results of the random-effect analysis of all the antihyperglycemic drugs compared with placebo and compared with one another. Compared with placebo, all the antihyperglycemic drugs included in the network had statistically significantly lower $\mathrm{A}_{1 \mathrm{C}}$ at follow-up. There were no statistically significant differences among Albi, Dula, and Lira, compared to EQW. Compared with EBID, Dula, EQW, and Lira had a significantly better effect on $A_{1 C}$. For the odds of reaching the $<7 \%$ target, all GLP-1 RAs in the network had significantly higher odds of reaching the $<7 \%$ target compared with placebo. Dula, EQW, and Lira had higher odds of reaching target compared to Albi. Lastly, the odds of reaching $<7 \%$ target were not significantly different between Albi and EBID (Table 2).

Table 3 shows the probability of reaching the target $<7 \%$ $\mathrm{A}_{1 \mathrm{C}}$, number needed to treat (NNT), number of patients reaching target per 100 treated, and absolute change in $\mathrm{A}_{1 \mathrm{C}}$ from base case for each treatment group across all analyses. NNT is the number needed to be treated in order to observe one event of interest, and the lower the NNT (closest to 1), the more effective the treatment. We estimate that for every two patients treated with a GLP-1 RA, one will meet the $<7 \% \mathrm{~A}_{1 \mathrm{C}}$ target within 6 months. Patients are more likely to reach the $<7 \%$ target at 6 months with Dula, EQW, and Lira compared to Albi. For every 100 patients treated, nine more will reach the $<7 \%$ target if treated with Dula, EQW, and Lira compared to Albi (Table 3).

Table I Summary of $A_{I C}$ end points from head-to-head GLP-I RA trials

\begin{tabular}{|c|c|c|c|c|c|}
\hline $\begin{array}{l}\text { Head-to-head } \\
\text { comparison }\end{array}$ & Study & $\begin{array}{l}\text { Reaching } A_{1 C} \text { target, } \\
\text { OR }(95 \% \mathrm{Cl})\end{array}$ & & WMD in $A_{1 C}$, OR $(95 \% \mathrm{Cl})$ & \\
\hline Dula versus EBID & AWARD-I ${ }^{42, *}$ & $2.35(1.73-3.19)$ & Dula better, $P<0.0$ I & $-0.4 I(-0.53$ to -0.3$)$ & Dula better, $P<0.0$ I \\
\hline \multirow[t]{2}{*}{ EQW versus EBID } & DURATION-I ${ }^{52}$ & $2.72(1.91-3.86)$ & EQW better, $P<0.01$ & $-0.55(-0.75$ to -0.35$)$ & QW better, $P<0.01$ \\
\hline & DURATION-5 $5^{56}$ & & & & \\
\hline Lira versus EBID & LEAD-6 63 & $1.57(1.09-2.27)$ & Lira better, $P=0.02$ & $-0.33(-0.55$ to -0.11$)$ & Lira better, $P<0.0 \mathrm{I}$ \\
\hline Albi versus Lira & HARMONY-7 ${ }^{41}$ & $0.68(0.52-0.9)$ & Lira better, $P<0.0 \mathrm{I}$ & $0.21(0.08-0.34)$ & Lira better, $P<0.0 \mathrm{I}$ \\
\hline Dula versus Lira & AWARD $-6^{47}$ & $1.02(0.72-1.44)$ & No difference, $P>0.05$ & $-0.06(-0.2$ to -0.08$)$ & No difference, $P>0.05$ \\
\hline EQW versus Lira & DURATION-6 $6^{57}$ & $0.74(0.57-0.96)$ & Lira better, $P=0.02$ & $0.2(0.06-0.34)$ & Lira better, $P<0.0 \mathrm{I}$ \\
\hline
\end{tabular}

Note: *Included a placebo arm.

Abbreviations: OR, odds ratio; $\mathrm{Cl}$, confidence interval; WMD, weighted mean difference; Dula, dulaglutide; EBID, exenatide bis in die (twice daily); EQW, exenatide quaque week (once weekly); Lira, liraglutide; Albi, albiglutide. 
Network 1: Analysis by drug (studies with 6-month follow-up)

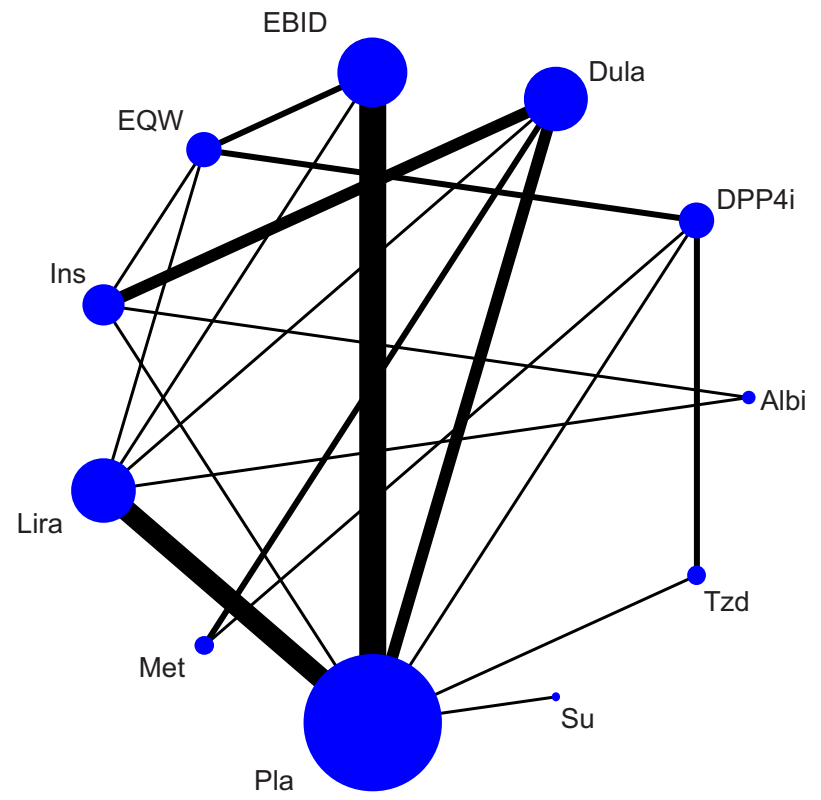

\begin{tabular}{|l|l|}
\hline Study arm & Pooled by drug \\
\hline Placebo & Pla \\
\hline Albiglutide $30 \mathrm{mg}$ once weekly & Albi \\
\hline Albiglutide $30-50 \mathrm{mg}$ once weekly & Albi \\
\hline Dulaglutide $0.75 \mathrm{mg}$ once weekly & Dula \\
\hline Dulaglutide $1.5 \mathrm{mg}$ once weekly & Dula \\
\hline Exenatide $10 \mu \mathrm{g}$ twice daily & EBID \\
\hline Exenatide $2 \mathrm{mg}$ once weekly & EQW \\
\hline Exenatide $5 \mu \mathrm{g}$ twice daily & EBID \\
\hline Glimepiride $4 \mathrm{mg}$ & Su \\
\hline Glimepiride $8 \mathrm{mg}$ & Su \\
\hline Insulin glargine (treat-to-target) & Ins \\
\hline Liraglutide $1.2 \mathrm{mg}$ once daily & Lira \\
\hline Liraglutide $1.8 \mathrm{mg}$ once daily & Lira \\
\hline Metformin $1.5-2 \mathrm{~g}$ & Met \\
\hline Pioglitazone $30-45$ mg once daily & Tzd \\
\hline Pioglitazone $45 \mathrm{mg}$ once daily & Tzd \\
\hline Rosiglitazone $4 \mathrm{mg} /$ day & Tzd \\
\hline Sitagliptin $100 \mathrm{mg}$ once daily & DPP4i \\
\hline
\end{tabular}

\section{Network 2: Analysis by drug (all studies)}

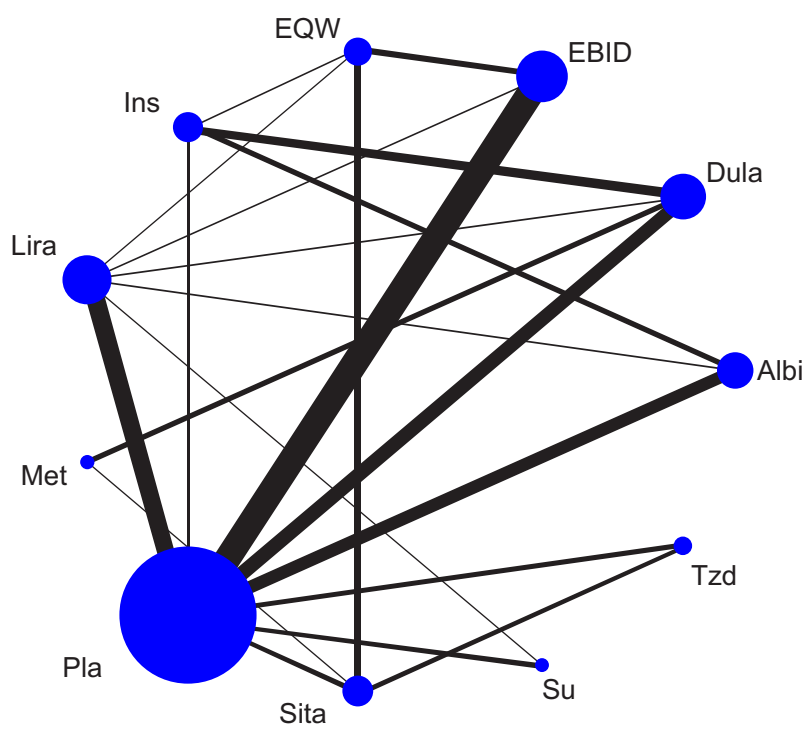

Figure I Network diagram for meta-analysis of $A_{1 C}$ outcomes.

Notes: Network 2 includes all 29 studies, network I (the base case) excludes LEAD-360 and HARMONY I-5 $5^{35-39}$ ( 23 studies); line thickness corresponds to number of study arms contributing to analysis.

Abbreviations: EBID, exenatide bis in die (twice daily); EQW, exenatide quaque week (once weekly); Dula, dulaglutide; Ins, insulin; Albi, albiglutide; Lira, liraglutide; Met, metformin; Tzd, thiazolidinedione; Pla, placebo; Su, sulfonylurea; DPP4i, DPP4 inhibitor; Sita, sitagliptin.

\section{Sensitivity analysis}

The base-case NMA excluded six studies (five studies for Albi and one for Lira) because they had longer follow-ups and did not report $\mathrm{A}_{1 \mathrm{C}}$ outcomes at 6 months. A sensitivity analysis was conducted to examine the impact of inclusion of these six studies on the relative treatment effects of GLP-1 RAs. This resulted in lower treatment effects for Albi compared with the base-case results, although the difference in effect was not significant (Figure 2). The treatment effects for the other GLP-1 RAs were largely unchanged between the 6-month base-case and sensitivity analyses. It was also noted that the inclusion of longer-term studies resulted in a lower treatment effect for sulfonylureas. This result would be due to the inclusion of the HARMONY-3 study, which included a glimepiride 
Table 2 Summary of 6-month network meta-analysis results

\begin{tabular}{|c|c|c|c|c|}
\hline \multirow[t]{2}{*}{ Comparison } & \multicolumn{2}{|c|}{ Reaching $<7 \%$ target, OR $(95 \% \mathrm{Crl})$} & \multicolumn{2}{|l|}{ WMD in $A_{I C},(95 \%$ Crl) } \\
\hline & RE base case & RE adjusted, $B L A_{I C}$ & RE base case & RE adjusted, $B L A_{I C}$ \\
\hline \multicolumn{5}{|l|}{ vs Pla } \\
\hline Albi & $3.852(2.224-6.733) *$ & $3.868(2.215-6.84 \mid)^{*}$ & $-0.949(-1.212 \text { to }-0.688)^{*}$ & $-0.949(-1.216 \text { to }-0.684)^{*}$ \\
\hline Dula & $5.455(3.97-7.558) *$ & $5.671(4.073-8.005)^{*}$ & $-1.097(-1.246 \text { to }-0.945)^{*}$ & $-1.106(-1.258 \text { to }-0.951)^{*}$ \\
\hline EBID & $2.882(2.149-3.942)^{*}$ & $2.886(2.144-3.947)^{*}$ & $-0.68(-0.813 \text { to }-0.547)^{*}$ & $-0.683(-0.816 \text { to }-0.549)^{*}$ \\
\hline EQW & $5.521(3.83-8.142)^{*}$ & $5.55 \mathrm{I}(3.83 \mathrm{I}-8.208)^{*}$ & $-1.042(-1.224 \text { to }-0.862)^{*}$ & $-1.047(-1.232 \text { to }-0.866)^{*}$ \\
\hline Lira & $5.437(4.063-7.359)^{*}$ & $5.344(3.956-7.261)^{*}$ & -1.131 (-1.277 to -0.988$)^{*}$ & $-1.124(-1.272 \text { to }-0.978)^{*}$ \\
\hline Su & $4.857(2.607-9.15)^{*}$ & $4.604(2.404-8.787)^{*}$ & -1.121 (-I.455 to -0.786$)^{*}$ & $-1.105(-1.445 \text { to }-0.765)^{*}$ \\
\hline Ins & $3.208(2.204-4.74)^{*}$ & $3.289(2.245-4.91)^{*}$ & $-0.82(-1.005 \text { to }-0.637)^{*}$ & $-0.827(-1.016 \text { to }-0.641)^{*}$ \\
\hline Met & $3.613(2.205-6.007)^{*}$ & $3.692(2.231-6.172)^{*}$ & $-0.889(-1.13 \text { to }-0.648)^{*}$ & $-0.897(-1.14 \mid \text { to }-0.65)^{*}$ \\
\hline Tzd & $3.46(2.229-5.456)^{*}$ & $3.442(2.207-5.461) *$ & -0.861 ( $(-1.094 \text { to }-0.618)^{*}$ & $-0.867(-1.102 \text { to }-0.62)^{*}$ \\
\hline DPP4i & $2.134(1.386-3.318)^{*}$ & $2.163(1.4-3.385)^{*}$ & $-0.556(-0.761 \text { to }-0.345)^{*}$ & $-0.568(-0.78 \mathrm{I} \text { to }-0.35 \mathrm{I})^{*}$ \\
\hline \multicolumn{5}{|l|}{ Covariate } \\
\hline \multicolumn{5}{|c|}{ GLP-I RAs head-to-head } \\
\hline \multicolumn{5}{|l|}{ Albi vs } \\
\hline Dula & $0.706(0.409-\mid .221)$ & $0.682(0.39-1.196)$ & $0.148(-0.113$ to 0.404$)$ & $0.157(-0.11$ to $0.4 \mid 8)$ \\
\hline EBID & $1.336(0.746-2.362)$ & $1.34 \mid(0.742-2.398)$ & $-0.268(-0.542$ to 0.003$)$ & $-0.265(-0.543$ to 0.009$)$ \\
\hline EQW & $0.698(0.39-1.232)$ & $0.697(0.386-1.248)$ & $0.093(-0.181$ to 0.368$)$ & $0.098(-0.18$ to 0.376$)$ \\
\hline Lira & $0.708(0.428-1.172)$ & $0.724(0.435-1.212)$ & $0.182(-0.056$ to $0.42 I)$ & $0.176(-0.068$ to 0.418$)$ \\
\hline \multicolumn{5}{|l|}{ Dula vs } \\
\hline Albi & $1.416(0.819-2.444)$ & $1.466(0.837-2.566)$ & $-0.148(-0.404$ to 0.113$)$ & $-0.157(-0.418$ to 0.11$)$ \\
\hline EBID & $1.893(1.322-2.683)^{++}$ & $1.965(1.356-2.829)^{++}$ & $-0.417(-0.582 \text { to }-0.25)^{++}$ & $-0.423(-0.589 \text { to }-0.255)^{++}$ \\
\hline EQW & $0.988(0.677-1.422)$ & I.023 (0.69I-I.49) & $-0.055(-0.231$ to 0.126$)$ & $-0.059(-0.236$ to 0.124$)$ \\
\hline Lira & $1.003(0.722-1.395)$ & $1.061(0.747-1.516)$ & $0.034(-0.122$ to 0.195$)$ & $0.019(-0.146$ to 0.187$)$ \\
\hline \multicolumn{5}{|l|}{ EQW vs } \\
\hline Albi & $\mathrm{I} .433(0.8 \mathrm{II}-2.565)$ & $\mathrm{I} .435(0.80 \mathrm{I}-2.59)$ & $-0.093(-0.368$ to 0.181$)$ & $-0.098(-0.376$ to 0.18$)$ \\
\hline Dula & $1.012(0.703-1.477)$ & $0.978(0.67|-| .448)$ & $0.055(-0.126$ to $0.23 \mathrm{I})$ & $0.059(-0.124$ to 0.236$)$ \\
\hline EBID & $1.915(1.337-2.766)^{++}$ & $1.924(1.33-2.79)^{++}$ & $-0.362(-0.543 \text { to }-0.184)^{++}$ & $-0.364(-0.547 \text { to }-0.185)^{++}$ \\
\hline Lira & $1.014(0.72-1.452)$ & $1.038(0.73-1.502)$ & $0.089(-0.086$ to 0.263$)$ & $0.077(-0.103$ to 0.256$)$ \\
\hline \multicolumn{5}{|l|}{ Lira vs } \\
\hline Albi & $1.412(0.854-2.334)$ & $1.38 \mid(0.825-2.298)$ & $-0.182(-0.421$ to 0.056$)$ & $-0.176(-0.418$ to 0.068$)$ \\
\hline Dula & $0.997(0.717-1.385)$ & $0.943(0.66-1.339)$ & $-0.034(-0.195$ to 0.122$)$ & $-0.019(-0.187$ to 0.146$)$ \\
\hline EBID & $1.887(1.338-2.638)^{++}$ & $1.853(1.302-2.605)^{++}$ & $-0.45 \mathrm{I}(-0.617 \text { to }-0.287)^{++}$ & $-0.441(-0.609 \text { to }-0.274)^{++}$ \\
\hline EQW & $0.986(0.689-1.388)$ & $0.964(0.666-1.37)$ & $-0.089(-0.263$ to 0.086$)$ & $-0.077(-0.256$ to 0.103$)$ \\
\hline
\end{tabular}

Notes: *Significantly better compared to placebo; ${ }^{++}$significantly better compared to the active control.

Abbreviations: OR, odds ratio; Crl, credible interval; WMD, weighted mean difference; RE, random-effect; $\mathrm{Bl}$, baseline; Pla, placebo; Albi, albiglutide; Dula, dulaglutide; EBID, exenatide bis in die (twice daily); EQW, exenatide quaque week (once weekly); Lira, liraglutide; Su, sulfonylurea; Ins, insulin; Met, metformin; Tzd, thiazolidinedione; DPP4i, DPP4 inhibitor; vs, versus.

Table 3 Probability of reaching $<7 \% A_{I C}$ target and absolute change in $A_{I C}$ at 6 months

\begin{tabular}{lllll}
\hline GLP-I RA & $<7 \% \mathbf{A}_{\text {IC }}$ target (range) & NNT* & NRT per I00 treated & $\begin{array}{l}\text { Absolute change in A } \\
\text { with baseline, } \text { OR (CI) }\end{array}$ \\
\hline Albi & $43.1 \%(26.4 \%-61.7 \%)$ & 2.32 & 43.1 & $-1.022(-1.334 \text { to }-0.709)^{\ddagger}$ \\
Dula & $52.7 \%(37.8 \%-67 \%)$ & 1.9 & 52.7 & $-1.178(-1.404 \text { to }-0.952)^{\ddagger}$ \\
EBID & $36.1 \%(24 \%-50.5 \%)$ & 2.77 & 36.1 & $-0.756(-0.969 \text { to }-0.543)^{\ddagger}$ \\
EQW & $52.1 \%(36.8 \%-67.3 \%)$ & 1.92 & 52.1 & $-1.12(-1.367 \text { to }-0.874)^{\ddagger}$ \\
Lira & $51.2 \%(36.9 \%-65.3 \%)$ & 1.96 & 51.2 & $-1.197(-1.418 \text { to }-0.977)^{\ddagger}$ \\
\hline
\end{tabular}

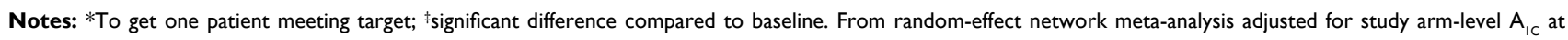
baseline.

Abbreviations: NNT, number needed to treat; NRT, number reaching target; OR, odds ratio; Cl, confidence interval; Albi, albiglutide; Dula, dulaglutide; EBID, exenatide bis in die (twice daily); EQW, exenatide quaque week (once weekly); Lira, liraglutide.

arm and reported end points after 104 weeks. Such a finding is consistent with other studies, in that although sulfonylurea treatment can result in a rapid initial response, the effectiveness diminishes over time, resulting in a gradual increase in $\mathrm{A}_{1 \mathrm{C}}{ }^{64-66}$
An additional covariate analysis (results not shown) was undertaken to take into account differences in the background treatment across trials. This incorporated two variables: the percentage of patients on an oral antidiabetes drug at baseline 


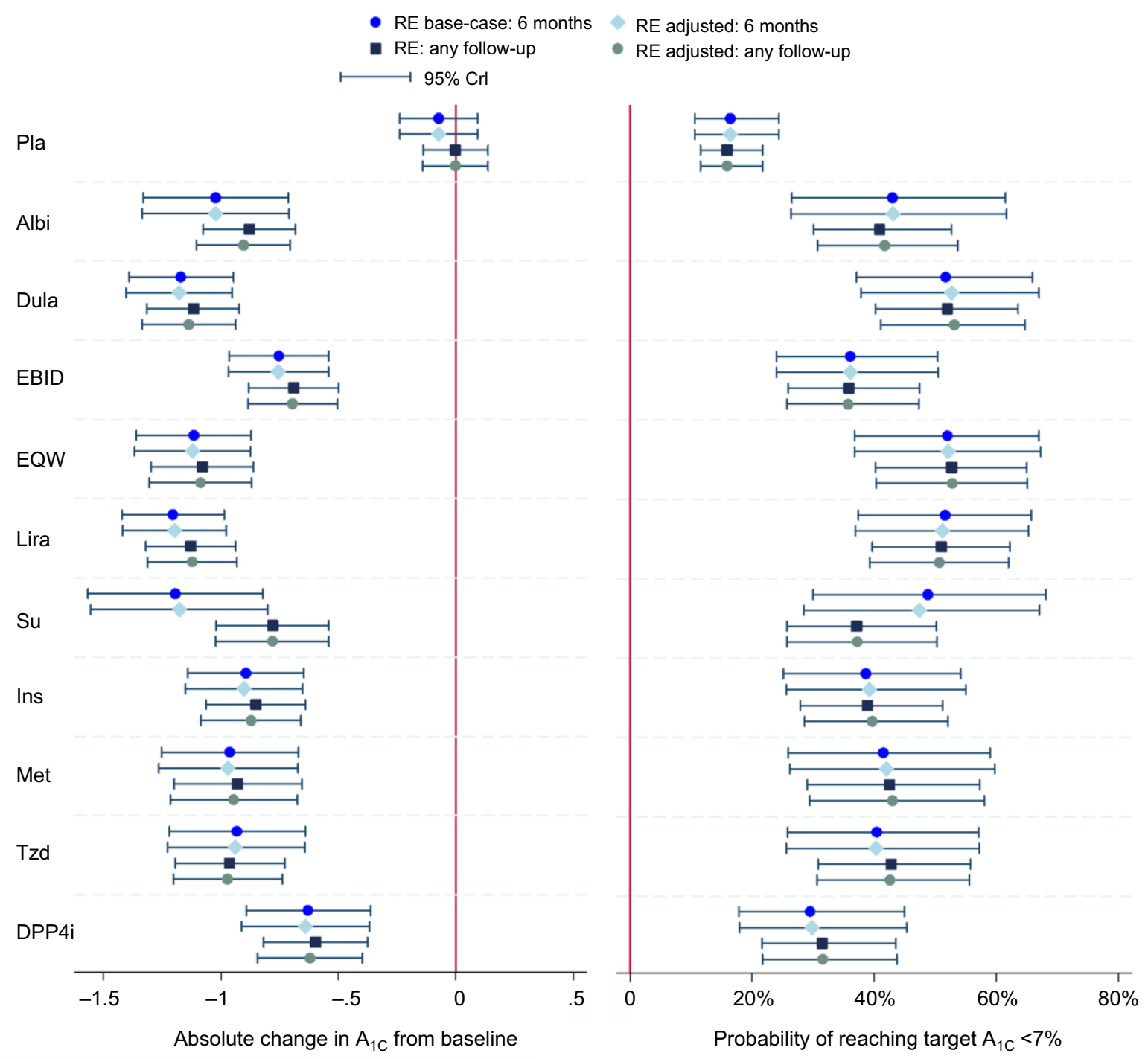

Figure 2 Summary of network meta-analysis results: absolute change in $A_{I C}$ and probability of reaching $<7 \%$ treatment target.

Notes: RE, random effect; Crl, credible interval; Pla, placebo; Albi, albiglutide; Dula, dulaglutide; EBID, exenatide bis in die (twice daily); EQW, exenatide quaque week (once weekly); Lira, liraglutide; Su, sulfonylurea; Ins, insulin; Met, metformin; Tzd, thiazolidinedione; DPP4i, dipeptidyl peptidase-4 inhibitor.

and the use of insulin as part of the background treatment. Use of a background oral antidiabetes drug was not a significant predictor of treatment effect. There were insufficient studies to assess whether background insulin could have been a potential effect modifier.

\section{Consistency between direct and indirect evidence}

When comparing the direct GLP-1 RA head-to-head results shown in Table 1 with the NMA results in Table 2, the results are largely consistent. The direct results comparing Dula, EQW, and Lira versus EBID had corresponding statistically significant results in the random-effect base-case NMA.

For the comparisons of Albi, Dula, and EQW versus Lira, the random-effect base-case NMA did not produce statistically significant results, although statistically significant differences $(P<0.05)$ were reported in HARMONY-7 (Lira better than Albi) and DURATION-6 (Lira better than EQW). It was noted that both HARMONY-7 and DURATION-6 had an open-label design and unmatched administration of study drugs (daily versus weekly).

In the course of conducting the analysis, it was noted that AWARD-1 and Moretto et $\mathrm{al}^{51}$ reported that the efficacy of 10 $\mu \mathrm{g}$ EBID was not significantly different from placebo (odds ratio versus placebo 1.46 , [95\% CI 0.94-2.26], and 2.14, [95\% CI 0.99-4.63], respectively). As the treatment-effect CI for Moretto et al overlapped the CIs estimated from the other three EBID trials (Figure 3 ) and given the study size (56 and 59 patients in the exenatide and placebo arms, respectively), we attribute the lack of significance to a lack of power to detect differences between study arms. AWARD-1, on the other hand, included sufficient numbers of patients to detect 


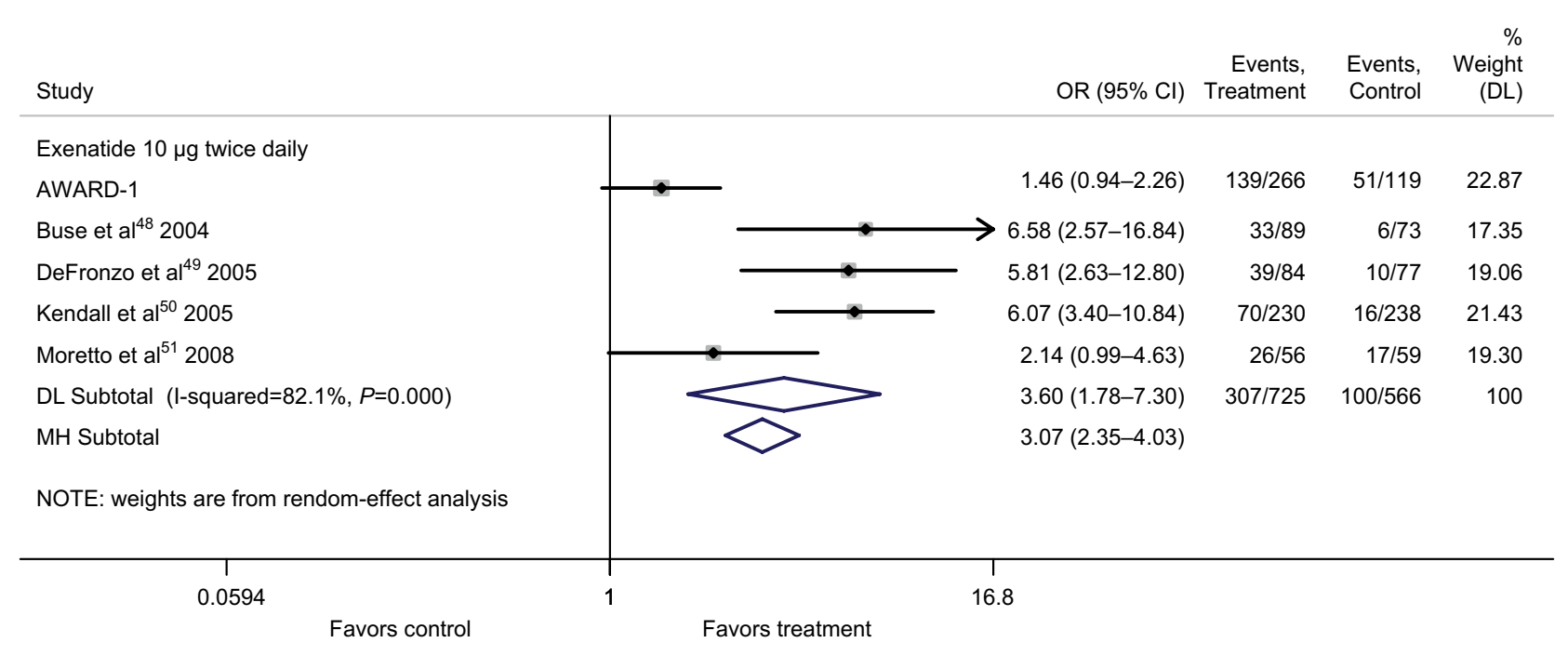

Figure 3 Direct meta-analysis results: odds of reaching $<7 \%$ treatment target for exenatide $10 \mu \mathrm{g}$ twice daily versus placebo.

Abbreviations: OR, odds ratio; Cl, confidence interval; DL, DerSimonian-Laird (random-effect model); MH, Mantel-Haenszel (fixed-effect model).

differences. The upper bound for the CI lay below the lower bound for the CIs estimated from Buse et al, ${ }^{48}$ DeFronzo et al, ${ }^{49}$ and Kendall et al ${ }^{50}$ (Figure 3). The exenatide result from AWARD-1 appears to be inconsistent with the other trials. One potential explanation for the difference in effects across these trials is lack of blinding for the EBID arm in AWARD-1: placebo was given QW to match the Dula arms, but there was no dummy-placebo injection to match the BID dosing for exenatide.

In HARMONY-2, it was noted that the $50 \mathrm{mg}$ Albi arm was not as effective as the $30 \mathrm{mg}$ Albi arm at achieving the $<7 \% \mathrm{~A}_{1 \mathrm{C}}$ target $(40.2 \%$ versus $49 \%$ ). Note that the data for the HARMONY-2 study were taken from the clinical trials registry, as the study was not published in full at the time of this analysis. There may be unreported factors that would explain this result, but our analysis reflects the data as they were reported at the time of writing.

\section{Discussion}

In this NMA, we combined direct and indirect evidence from 29 RCTs involving 18,542 patients with T2D to estimate the relative efficacy among licensed GLP-1 RAs on the gold standard measure of diabetes control $-\mathrm{A}_{1 \mathrm{C}}$. We made several key observations: 1) GLP-1 RAs were superior to placebo in improving $A_{1 C}$, with moderate confidence in estimates; 2) relative efficacy was similar among longer-acting GLP-1 RAs, and absolute reduction in $\mathrm{A}_{1 \mathrm{C}}$ at 6 months was consistent among Dula, EQW, and Lira and was estimated to be within the range $0.9 \%-1.4 \%$; and 3 ) using NNT, we estimated that for every two patients treated with a GLP-1 RA, one will meet the $<7 \% \mathrm{~A}_{1 \mathrm{C}}$ target within 6 months.

Compared to direct evidence from head-to-head studies, evidence generated from this NMA allows for a more accurate assessment of GLP-1 RA relative efficacy on a class-wide level, which is especially important for population-health decision makers. ${ }^{21}$ Although direct evidence can provide health care decision makers with a crude sense of relative efficacy, such comparisons often lack details on the relative magnitude of treatment effects, are biased due to open-label trial design, and lack statistical power due to small sample size. The inconsistent results yielded from numerous direct comparisons also make it difficult for health care decision makers to reach definitive conclusions on a treatment decision.

While NMAs are useful in quantifying treatment effects from clinical studies, meta-analysis results can vary depending on the trials included in the network, the statistical methods applied, and the consideration of covariates to account for trial-design differences. Our network analysis was a comprehensive analysis of the $\mathrm{A}_{1 \mathrm{C}}$ outcome across all US-licensed GLP-1 RAs. Our results are corroborated by other published meta-analyses of GLP-1 RAs, which showed that patients with $T 2 \mathrm{D}$ can expect to improve their $\mathrm{A}_{1 \mathrm{C}}$ with GLP-1 RA therapy. ${ }^{14,16,19}$ However, our analysis completes the evidence for GLP-1 RAs, since other analyses either did not cover all currently available GLP-1 RAs in the US, ${ }^{13-17,19}$ limited study inclusion to placebo or specific active controls or combinations, ${ }^{14,16,17}$ or did not conduct an NMA to provide 
comparisons of all GLP-1 RAs against one another. ${ }^{13,15-17}$ Furthermore, some studies did not use an established NMA method, such as the Bayesian method recommended by the NICE Decision Support Unit, ${ }^{21}$ and/or the NMA did not control for study-arm baseline $\mathrm{A}_{1 \mathrm{C}}$ which varied across RCTs. ${ }^{19}$ Although baseline $A_{1 C}$ was not found to be a statistically significant covariate in this study, other studies have shown that baseline $A_{1 C}$ value could impact the magnitude of $A_{1 C}$ reduction where higher reductions are associated with higher baseline $\mathrm{A}_{1 \mathrm{C}}$ values. ${ }^{32}$

The implications of consistent glycemic control help clinicians design individualized treatment plans. While new drug therapies target the multiple defects that together contribute to diabetes, the possibility of improved control through GLP-1 RAs may lead to improved patient outcomes beyond glycemic control. American Association of Clinical Endocrinologists guidelines recommend initiating treatment with metformin in patients with entry $\mathrm{A}_{1 \mathrm{C}}>7.5 \%$ plus a second agent, with preference given to treatments with low potential for hypoglycemia and weight-loss effects. ${ }^{67}$ Therefore, GLP-1 RAs are ranked hierarchically first before other options in this regard. In addition, increased $\mathrm{A}_{1 \mathrm{C}}$ has been associated with microvascular and macrovascular complications, and lowering $\mathrm{A}_{1 \mathrm{C}}$ to below or around $7 \%$ has been shown to reduce microvascular and neuropathic complications of T1D and T2D. ${ }^{68,69}$ Most recently, this class has been shown to reduce major adverse cardiac events in large cardiovascular-outcome trials. Altogether, these findings, along with the known glycemic effects and beneficial secondary effects, may compel clinicians to use these agents in patients requiring robust and sustained control of their hyperglycemia, while addressing concerns of weight gain and hypoglycemia typically seen with traditional agents.

While important, the impact of a GLP-1 RA on $A_{1 C}$ reduction is just one consideration when selecting the best treatment for a patient. Karagiannis et $\mathrm{al}^{16}$ provided a direct meta-analysis of weekly GLP-1 RAs that also covered other important outcomes, such as weight change and gastrointestinal and injection-site reactions. However, the analysis did not provide head-to-head or indirect comparisons, and there were insufficient results for EQW, which had a large clinical program design comparable with others in the class. A recent NMA by Sun et al ${ }^{18}$ focused on gastrointestinal adverse events, specifically nausea, vomiting, and diarrhea, and indicated that these effects are associated with GLP-1 RAs. More recently, Zaccardi et al ${ }^{19}$ performed an NMA of weekly GLP-1 RAs using the frequentist approach, and reported no differences between EQW and a maintenance dose of Dula
(1.5 mg) for $\mathrm{A}_{1 \mathrm{C}}$ or on all three metabolic outcomes (blood pressure, blood lipids, and C-reactive protein), and both treatments reduced $\mathrm{A}_{1 \mathrm{C}}$ to a greater extent than albiglutide.

Managing blood glucose is fundamental to caring for people with T2D. With newer glucose-dependent agents, such as GLP-1 RAs, CER is an increasingly important tool, given the wide range of treatment options available in each class. It allows health care decision makers to evaluate the efficacy and safety of multiple treatment options simultaneously. Such methods as NMA have been well recognized as useful tools to evaluate the relative merits of treatments when direct head-to-head studies are not available.

\section{Limitations}

In this comparative-effectiveness analysis, the NMA method was used to integrate placebo- and active-controlled trial data to assess the relative efficacy of US-approved GLP-1 RAs. The NMA approach has an advantage in that it preserves randomized comparisons and gives each trial an appropriate weighting, while including data from both direct (head-tohead) studies and indirect studies (eg, via placebo). Inferences that are based on the direct evidence alone ignore a substantial part of the available clinical evidence. An NMA that includes both direct and indirect evidence provides a more comprehensive assessment of efficacy, and is less prone to study-selection bias. The value of NMA to CER is that it allows us to assess the magnitude of an intervention's effect and its consistency across trials, as opposed to a "votecounting" approach, which infers the presence, or not, of an effect based on the statistical significance of results in each study. ${ }^{30,70}$ Despite its strengths, this method is not without challenges. ${ }^{71,72}$ Therefore, our findings should be interpreted in light of the following limitations. One criticism of NMAs is that the methodology can lack transparency and results can be difficult to reproduce. Recent guidelines have helped standardize methods to improve confidence in NMAs. ${ }^{73}$ For our analysis, we followed the NICE Decision Support Unit recommendations that were developed in collaboration with leading academics for conducting and reporting NMAs, and used validated code that is available in the public domain. ${ }^{21}$

Another limitation is that the NMAs rely on the assumption that data are consistent across trials. However, this is a problem associated with data synthesis in general and not just (network) meta-analysis. Problems with consistency may arise if the inclusion criteria are too broad, such that the trial populations are not comparable clinically. For example, treatment-naïve patients may have a higher response to treatment compared with patients for whom one or more lines of 
treatment have failed. There may also be undetected heterogeneity across trials that may arise from study bias, eg, poor quality, or small study bias where the trial results appear to be outliers. Some heterogeneity is to be expected, and some differences across clinical studies may reflect differences in real-world practice. There may be imbalances in the distribution of unobserved or unmeasured effect modifiers that have the potential to confound the comparative estimates among GLP-1 RAs. Also, we did not consider whether differences between safety outcomes across GLP-1 RAs impact on treatment efficacy, though this has been considered elsewhere. ${ }^{18}$

Finally, our NMA included non-GLP-1 RA drugs (eg, metformin, sulfonylureas, insulin) as control arms or additional arms from the GLP-1 RA trials. These arms are required to connect the network of evidence across the GLP-1 RA trials that are the focus of this analysis. While our analysis suggests lower relative efficacy of non-GLP-1 RAs versus GLP-1 RAs, the analysis does not include all available evidence for the non-GLP-1 RA drugs.

\section{Conclusion}

This is a comprehensive assessment of the comparative effectiveness of US-licensed GLP-1 RAs in terms of $A_{1 C}$. GLP-1 RAs are superior to placebo in improving glycemic control, with a consistent absolute reduction in $\mathrm{A}_{1 \mathrm{C}}$ at 6 months, ranging from $0.9 \%$ to $1.4 \%$, among Dula, EQW, and Lira. In terms of NNT, we estimate that for every two patients treated with a GLP-1 RA, one will meet the $<7 \% \mathrm{~A}_{1 \mathrm{C}}$ target within 6 months of commencing GLP-1 RAs. These GLP-1 RAs in particular should thus be considered a viable addition to oral antidiabetes therapy in the appropriate patient.

\section{Disclosure}

MEO received funding from AstraZeneca US, and HN, JL, and SAT are paid employees of AstraZeneca US. The authors report no other conflicts of interest in this work.

\section{References}

1. Handelsman Y, Bloomgarden ZT, Grunberger G, et al. American association of clinical endocrinologists and American college of endocrinology - clinical practice guidelines for developing a diabetes mellitus comprehensive care plan - 2015. Endocr Pract. 2015;21(Suppl 1):1-87.

2. Inzucchi SE, Bergenstal RM, Buse JB, et al. Management of hyperglycemia in type 2 diabetes, 2015 - a patient-centered approach: update to a position statement of the American Diabetes Association and the European Association for the Study of Diabetes. Diabetes Care. 2015;38(1):140-149.

3. Canadian Diabetes Association Clinical Practice Guidelines Expert C, Imran SA, Rabasa-Lhoret R, Ross S. Targets for glycemic control. Can J Diabetes. 2013;37(Suppl 1):S31-S34.

4. International Diabetes Federation, Clinical Guidelines Task Force. Global guideline for type 2 diabetes. Available from http://www.idf. org/guideline-type-2-diabete. Accessed October 2015.
5. Internal Clinical Guidelines Team, National Institute for Health and Care Excellence. Clinical guideline update. Type 2 diabetes in adults: management. Available from http://www.nice.org.uk/guidance/indevelopment/gid-cgwave0612/documents. Accessed October 2015.

6. Garber AJ, Abrahamson MJ, Barzilay JI, et al. Consensus statement by the American Association of Clinical Endocrinologists and American College of Endocrinology on the comprehensive type 2 diabetes management algorithm: 2016 executive summary. Endocr Pract. 2016;22(1):84-113.

7. Drucker DJ, Nauck MA. The incretin system: glucagon-like peptide-1 receptor agonists and dipeptidyl peptidase-4 inhibitors in type 2 diabetes. Lancet. 2006;368(9548):1696-1705.

8. Bucher HC, Guyatt GH, Griffith LE, Walter SD. The results of direct and indirect treatment comparisons in meta-analysis of randomized controlled trials. J Clin Epidemiol. 1997;50(6):683-691.

9. Glenny AM, Altman DG, Song F, et al. Indirect comparisons of competing interventions. Health Technol Assess. 2005;9(26):1-134, iii-iv.

10. Caldwell DM, Ades AE, Higgins JP. Simultaneous comparison of multiple treatments: combining direct and indirect evidence. $B M J$. 2005;331(7521):897-900.

11. Sutton A, Ades AE, Cooper N, Abrams K. Use of indirect and mixed treatment comparisons for technology assessment. Pharmacoeconomics. 2008;26(9):753-767.

12. Lu G, Ades AE. Combination of direct and indirect evidence in mixed treatment comparisons. Stat Med. 2004;23(20):3105-3124.

13. Aroda VR, Henry RR, Han J, et al. Efficacy of GLP-1 receptor agonists and DPP-4 inhibitors: meta-analysis and systematic review. Clin Ther. 2012;34(6):1247-1258.e22.

14. Scott DA, Boye KS, Timlin L, Clark JF, Best JH. A network metaanalysis to compare glycaemic control in patients with type 2 diabetes treated with exenatide once weekly or liraglutide once daily in comparison with insulin glargine, exenatide twice daily or placebo. Diabetes Obes Metab. 2013;15(3):213-223.

15. Eng C, Kramer CK, Zinman B, Retnakaran R. Glucagon-like peptide-1 receptor agonist and basal insulin combination treatment for the management of type 2 diabetes: a systematic review and meta-analysis. Lancet. 2014;384(9961):2228-2234.

16. Karagiannis T, Liakos A, Bekiari E, et al. Efficacy and safety of once-weekly glucagon-like peptide 1 receptor agonists for the management of type 2 diabetes: a systematic review and meta-analysis of randomized controlled trials. Diabetes Obes Metab. 2015;17(11): 1065-1074.

17. Liu FP, Dong JJ, Yang Q, et al. Glucagon-like peptide 1 receptor agonist therapy is more efficacious than insulin glargine for poorly controlled type 2 diabetes: a systematic review and meta-analysis. $J$ Diabetes. 2015;7(3):322-328.

18. Sun F, Chai S, Yu K, et al. Gastrointestinal adverse events of glucagonlike peptide- 1 receptor agonists in patients with type 2 diabetes: a systematic review and network meta-analysis. Diabetes Technol Ther. 2015;17(1):35-42.

19. Zaccardi F, Htike ZZ, Webb DR, Khunti K, Davies MJ. Benefits and harms of once-weekly glucagon-like peptide-1 receptor agonist treatments: a systematic review and network meta-analysis. Ann Intern Med. 2016;164(2):102-113.

20. ClinicalTrials.gov [homepage on the Internet]. Available from: https:// clinicaltrials.gov. Accessed April 15, 2015.

21. Dias S, Welton NJ, Sutton AJ, Ades AE. NICE DSU Technical Support Document 2: A Generalised Linear Modelling Framework for Pairwise and Network Meta-analysis of Randomised Controlled Trials. Sheffield: Decision Support Unit; 2011.

22. Ades AE, Sculpher M, Sutton A, et al. Bayesian methods for evidence synthesis in cost-effectiveness analysis. Pharmacoeconomics. 2006;24(1):1-19.

23. Sutton A, Ades AE, Cooper N, Abrams K. Use of indirect and mixed treatment comparisons for technology assessment. Pharmacoeconomics. 2008;26(9):753-767.

24. Sutton AJ, Abrams KR. Bayesian methods in meta-analysis and evidence synthesis. Stat Methods Med Res. 2001;10(4):277-303. 
25. MRC Biostatistics Unit. The BUGS project. Available from: http://www. mrc-bsu.cam.ac.uk/bugs. Accessed August 19, 2016.

26. Lunn D, Jackson C, Best N, Thomas A, Spiegelhalter D. The BUGS Book: A Practical Introduction to Bayesian Analysis. London: CRC Press; 2013.

27. MRC Biostatistics Unit. DIC: deviance information criterion. Available from: http://www.mrc-bsu.cam.ac.uk/bugs/winbugs/dicpage.shtml\#q15. Accessed August 19, 2016.

28. DerSimonian R, Laird N. Meta-analysis in clinical trials. Control Clin Trials. 1986;7(3):177-188.

29. Fleiss JL. The statistical basis of meta-analysis. Stat Methods Med Res. 1993;2(2):121-145.

30. Borenstein M, Hedges LV, Higgins JP, Rothstein HR. Introduction to Meta-analysis. Chichester, UK: Wiley \& Sons; 2009.

31. Dias S, Sutton AJ, Welton NJ, Ades AE. NICE DSU Technical Support Document 3 - Heterogeneity: Subgroups, Meta-regression, Bias and Bias-Adjustment. Sheffield: Decision Support Unit; 2011.

32. DeFronzo RA, Stonehouse AH, Han J, Wintle ME. Relationship of baseline $\mathrm{HbA} 1 \mathrm{c}$ and efficacy of current glucose-lowering therapies: a metaanalysis of randomized clinical trials. Diabet Med.2010;27(3):309-317.

33. StataCorp LP. Stata/MP 14.0 for Windows [software]. Available from www.stata.com/statamp. Accessed August 19, 2016.

34. Harris RJ, Bradburn MJ, Deeks JJ, Harbord RM, Altman DG, Sterne JA. Metan: fixed- and random-effects meta-analysis. Stata J. 2008;8(1):3-28.

35. Reusch J, Stewart MW, Perkins CM, et al. Efficacy and safety of onceweekly glucagon-like peptide 1 receptor agonist albiglutide (HARMONY 1 trial): 52-week primary endpoint results from a randomized, double-blind, placebo-controlled trial in patients with type 2 diabetes mellitus not controlled on pioglitazone, with or without metformin Diabetes Obes Metab. 2014;16(12):1257-1264.

36. GlaxoSmithKline. Safety and efficacy study of albiglutide in type 2 diabetes. Available from: https://clinicaltrials.gov/ct2/show/NCT00849017. NLM identifier: NCT00849017.Accessed August 19, 2016.

37. Ahren B, Johnson SL, Stewart M, et al. HARMONY 3: 104-week randomized, double-blind, placebo- and active-controlled trial assessing the efficacy and safety of albiglutide compared with placebo, sitagliptin, and glimepiride in patients with type 2 diabetes taking metformin. Diabetes Care. 2014;37(8):2141-2148.

38. Weissman PN, Carr MC, Ye J, et al. HARMONY 4: randomised clinical trial comparing once-weekly albiglutide and insulin glargine in patients with type 2 diabetes inadequately controlled with metformin with or without sulfonylurea. Diabetologia. 2014;57(12):2475-2484.

39. Home PD, Shamanna P, Stewart M, et al. Efficacy and tolerability of albiglutide versus placebo or pioglitazone over 1 year in people with type 2 diabetes currently taking metformin and glimepiride: HARMONY 5. Diabetes Obes Metab. 2015;17(2):179-187.

40. Rosenstock J, Fonseca VA, Gross JL, et al. Advancing basal insulin replacement in type 2 diabetes inadequately controlled with insulin glargine plus oral agents: a comparison of adding albiglutide, a weekly GLP-1 receptor agonist, versus thrice-daily prandial insulin lispro. Diabetes Care. 2014;37(8):2317-2325.

41. Pratley RE, Nauck MA, Barnett AH, et al. Once-weekly albiglutide versus once-daily liraglutide in patients with type 2 diabetes inadequately controlled on oral drugs (HARMONY 7): a randomised, open-label, multicentre, non-inferiority phase 3 study. Lancet Diabetes Endocrinol. 2014;2(4):289-297.

42. Wysham C, Blevins T, Arakaki R, et al. Efficacy and safety of dulaglutide added onto pioglitazone and metformin versus exenatide in type 2 diabetes in a randomized controlled trial (AWARD-1). Diabetes Care. 2014;37(8):2159-2167.

43. Giorgino F, Benroubi M, Sun JH, Zimmermann AG, Pechtner V. Efficacy and safety of once-weekly dulaglutide versus insulin glargine in patients with type 2 diabetes on metformin and glimepiride (AWARD-2). Diabetes Care. 2015;38(12):2241-2249.

44. Umpierrez G, Povedano ST, Manghi FP, Shurzinske L, Pechtner V. Efficacy and safety of dulaglutide monotherapy versus metformin in type 2 diabetes in a randomized controlled trial (AWARD-3). Diabetes Care. 2014;37(8):2168-2176.
45. Blonde L, Jendle J, Gross J, et al. Once-weekly dulaglutide versus bedtime insulin glargine, both in combination with prandial insulin lispro, in patients with type 2 diabetes (AWARD-4): a randomised, open-label, phase 3, non-inferiority study. Lancet. 2015;385(9982): 2057-2066.

46. Nauck M, Weinstock RS, Umpierrez GE, Guerci B, Skrivanek Z, Milicevic Z. Efficacy and safety of dulaglutide versus sitagliptin after 52 weeks in type 2 diabetes in a randomized controlled trial (AWARD5). Diabetes Care. 2014;37(8):2149-2158.

47. Dungan KM, Povedano ST, Forst T, et al. Once-weekly dulaglutide versus once-daily liraglutide in metformin-treated patients with type 2 diabetes (AWARD-6): a randomised, open-label, phase 3, non-inferiority trial. Lancet. 2014;384(9951):1349-1357.

48. Buse JB, Henry RR, Han J, et al. Effects of exenatide (exendin-4) on glycemic control over 30 weeks in sulfonylurea-treated patients with type 2 diabetes. Diabetes Care. 2004;27(11):2628-2635.

49. DeFronzo RA, Ratner RE, Han J, Kim DD, Fineman MS, Baron AD. Effects of exenatide (exendin-4) on glycemic control and weight over 30 weeks in metformin-treated patients with type 2 diabetes. Diabetes Care. 2005;28(5):1092-1100.

50. Kendall DM, Riddle MC, Rosenstock J, et al. Effects of exenatide (exendin-4) on glycemic control over 30 weeks in patients with type 2 diabetes treated with metformin and a sulfonylurea. Diabetes Care. 2005;28(5):1083-1091.

51. Moretto TJ, Milton DR, Ridge TD, et al. Efficacy and tolerability of exenatide monotherapy over 24 weeks in antidiabetic drug-naive patients with type 2 diabetes: a randomized, double-blind, placebo-controlled, parallel-group study. Clin Ther. 2008;30(8):1448-1460.

52. Drucker DJ, Buse JB, Taylor K, et al. Exenatide once weekly versus twice daily for the treatment of type 2 diabetes: a randomised, openlabel, non-inferiority study. Lancet. 2008;372(9645):1240-1250.

53. Bergenstal RM, Wysham C, Macconell L, et al. Efficacy and safety of exenatide once weekly versus sitagliptin or pioglitazone as an adjunct to metformin for treatment of type 2 diabetes (DURATION-2): a randomised trial. Lancet. 2010;376(9739):431-439.

54. Diamant M, Van Gaal L, Stranks S, et al. Once weekly exenatide compared with insulin glargine titrated to target in patients with type 2 diabetes (DURATION-3): an open-label randomised trial. Lancet. 2010;375(9733):2234-2243.

55. Russell-Jones D, Cuddihy RM, Hanefeld M, et al. Efficacy and safety of exenatide once weekly versus metformin, pioglitazone, and sitagliptin used as monotherapy in drug-naive patients with type 2 diabetes (DURATION-4): a 26-week double-blind study. Diabetes Care. 2012;35(2):252-258.

56. Blevins T, Pullman J, Malloy J, et al. DURATION-5: exenatide once weekly resulted in greater improvements in glycemic control compared with exenatide twice daily in patients with type 2 diabetes. $J$ Clin Endocrinol Metab. 2011;96(5):1301-1310.

57. Buse JB, Nauck M, Forst T, et al. Exenatide once weekly versus liraglutide once daily in patients with type 2 diabetes (DURATION-6): a randomised, open-label study. Lancet. 2013;381(9861):117-124.

58. Marre M, Shaw J, Brändle M, et al. Liraglutide, a once-daily human GLP-1 analogue, added to a sulphonylurea over 26 weeks produces greater improvements in glycaemic and weight control compared with adding rosiglitazone or placebo in subjects with type 2 diabetes (LEAD-1 SU). Diabet Med. 2009;26(3):268-278.

59. Nauck M, Frid A, Hermansen K, et al. Efficacy and safety comparison of liraglutide, glimepiride, and placebo, all in combination with metformin, in type 2 diabetes: the LEAD (liraglutide effect and action in diabetes)-2 study. Diabetes Care. 2009;32(1):84-90.

60. Garber A, Henry R, Ratner R, et al. Liraglutide versus glimepiride monotherapy for type 2 diabetes (LEAD-3 Mono): a randomised, 52-week, phase III, double-blind, parallel-treatment trial. Lancet. 2009;373(9662):473-481.

61. Zinman B, Gerich J, Buse JB, et al. Efficacy and safety of the human glucagon-like peptide-1 analog liraglutide in combination with metformin and thiazolidinedione in patients with type 2 diabetes (LEAD-4 Met+TZD). Diabetes Care. 2009;32(7):1224-1230. 
62. Russell-Jones D, Vaag A, Schmitz O, et al. Liraglutide vs insulin glargine and placebo in combination with metformin and sulfonylurea therapy in type 2 diabetes mellitus (LEAD-5 Met+SU): a randomised controlled trial. Diabetologia. 2009;52(10):2046-2055.

63. Buse JB, Rosenstock J, Sesti G, et al. Liraglutide once a day versus exenatide twice a day for type 2 diabetes: a 26-week randomised, parallel-group, multinational, open-label trial (LEAD-6). Lancet. 2009;374(9683):39-47.

64. Kahn SE, Haffner SM, Heise MA, et al. Glycemic durability of rosiglitazone, metformin, or glyburide monotherapy. $N$ Engl J Med. 2006;355(23):2427-2443.

65. Nauck MA, Del Prato S, Meier JJ, et al. Dapagliflozin versus glipizide as add-on therapy in patients with type 2 diabetes who have inadequate glycemic control with metformin: a randomized, 52-week, double-blind, active-controlled noninferiority trial. Diabetes Care. 2011;34(9):2015-2022.

66. Nauck MA, Meininger G, Sheng D, Terranella L, Stein PP. Efficacy and safety of the dipeptidyl peptidase-4 inhibitor, sitagliptin, compared with the sulfonylurea, glipizide, in patients with type 2 diabetes inadequately controlled on metformin alone: a randomized, double-blind, non-inferiority trial. Diabetes Obes Metab. 2007;9(2):194-205.

67. Garber AJ, Abrahamson MJ, Barzilay JI, et al. AACE/ACE comprehensive diabetes management algorithm 2015. Endocr Pract. 2015; 21(4):438-447.
68. Nathan DM, Buse JB, Davidson MB, et al. Medical management of hyperglycemia in type 2 diabetes - a consensus algorithm for the initiation and adjustment of therapy: a consensus statement of the American Diabetes Association and the European Association for the Study of Diabetes. Diabetes Care. 2009;32(1):193-203.

69. Stratton IM, Adler AI, Neil HA, et al. Association of glycaemia with macrovascular and microvascular complications of type 2 diabetes (UKPDS 35): prospective observational study. BMJ. 2000;321(7258):405-412.

70. Conn VS, Ruppar TM, Phillips LJ, Chase JA. Using meta-analyses for comparative effectiveness research. Nurs Outlook. 2012;60(4):182-190.

71. Cipriani A, Higgins JP, Geddes JR, Salanti G. Conceptual and technical challenges in network meta-analysis. Ann Intern Med. 2013;159(2): 130-137.

72. Mills EJ, Thorlund K, Ioannidis JP. Demystifying trial networks and network meta-analysis. BMJ. 2013;346:f2914.

73. Jansen JP, Fleurence R, Devine B, et al. Interpreting indirect treatment comparisons and network meta-analysis for health-care decision making: report of the ISPOR Task Force on Indirect Treatment Comparisons Good Research Practices - part 1. Value Health. 2011;14(4): $417-428$.

74. GlaxoSmithKline. Efficacy and safety of albiglutide in treatment of type 2 diabetes. Available from: https://clinicaltrials.gov/ct2/show/results/ NCT00838903. NLM identifier: NCT00838903. Accessed August 19, 2016 .
Diabetes, Metabolic Syndrome and Obesity: Targets and Therapy is an international, peer-reviewed open-access journal committed to the rapid publication of the latest laboratory and clinical findings in the fields of diabetes, metabolic syndrome and obesity research. Original research, review, case reports, hypothesis formation, expert opinion and commentaries are all considered for publication. The manuscript management system is completely online and includes a very quick and fair peer-review system, which is all easy to use. Visit http://www.dovepress.com/testimonials.php to read real quotes from published authors. 